\title{
Response production factors and reaction time
}

\author{
LES G. CARLTON and K. M. NEWELL \\ University of Illinois at Urbana-Champaign, Urbana, Illinois
}

\begin{abstract}
The relationship between reaction time and the force production characteristics of discrete responses was examined. Subjects produced isometric responses that varied with respect to peak force and time to peak force. Peak force varied from $5 \%$ to $90 \%$ of the subject's maximum force capabilities at three different time to peak force values $(100,200$, and $400 \mathrm{msec})$. The findings suggest that reaction time is independent of response duration and decreases at a negatively accelerating rate with increments of rate of force production.
\end{abstract}

The relationship between reaction time and the nature of the response to be produced has been of theoretical interest at least since DeLabarre, Logan, and Reed's (1897) investigation. Recent theorizing has centered around issues related to movement programming, with reaction time representing the time required by central processes to organize or program parameters for the to-be-produced response. Henry and Rogers (1960) proposed a memory drum model of response programming based on a computer analogy of how stored computer programs are accessed and run off. Henry and Rogers argued that as response complexity increased, reaction time also increased due to increased organizational demands. A number of variations on this original theme have emerged (e.g., Klapp, 1977; Kornblum \& Requin, 1984; Sternberg, Monsell, Knoll, \& Wright, 1978), in addition to an elaboration of the original memory drum model (Henry, 1981).

One of the most consistent findings about the relationships between reaction time and response parameters has been that reaction time increases as movement duration increases (e.g., Klapp, 1977; Klapp, McRae, \& Long, 1978; Klapp, Wyatt, \& Lingo, 1974; Zelaznik, Shapiro, \& Carter, 1982). Changes in reaction time as a function of response characteristics have been demonstrated in various types of discrete responses (e.g., Klapp \& Erwin, 1976; Zelaznik et al., 1982); the most systematic work has been done by Klapp and colleagues using keypress and discrete arm responses. However, other movementor force-related parameters vary with manipulations of response duration, such as the average velocity of the movement or the rate of force production in isometric tasks (Falkenberg \& Newell, 1980). Indeed, reaction time latencies have been found to be reduced even when movement duration is controlled and the velocity of the response is increased by increasing movement amplitude (M. J. Carlton, Robertson, L. G. Carlton, \& Newell, 1985;

This work was supported in part by Grant NSF BNS-83-17691 awarded to K. M. Newell. Requests for reprints should be sent to L. G. Carlton, Louise Freer Hall, University of Illinois, 906 S. Goodwin Ave., Urbana, IL 61801 .
Falkenberg \& Newell, 1980). This finding suggests that movement duration may not be an essential parameter in response organization, but rather may be an emergent property of the dynamic properties of the to-be-produced response.

Relating reaction time to the dynamic properties of movement production was the approach taken by DeLabarre et al. (1897), but limitations in their experimental design led to equivocal results, allowing Woodworth (1938) to conclude that there was no relationship between reaction time and force-time properties of the movement to be produced. Although a few studies have examined the relevance of response dynamics to reaction time (Ghez \& Vicario,1978; Klemmer, 1957; Nagasaki, Aoki, \& Nakamura, 1983), a consistent function has not emerged. However, recent evidence from our laboratory (L. G. Carlton, M. J. Carlton, \& Newell, 1987) has implicated the rate of force production as providing a systematic relationship between reaction time and movement parameters, with reaction time decreasing at a negatively accelerating rate with increments in rate of force production.

The experiment reported here provides a more comprehensive test of the relationship between reaction time and response dynamics. The experimental strategy was to manipulate the rate of force production by having subjects produce isometric responses with specific force-time profiles. Response duration, as represented by the duration of force production, and force level were manipulated across a wide range of conditions. Response durations of 200,400 , and 800 msec (times to peak force of 100,200 , and $400 \mathrm{msec}$ ) were combined with eight peak force levels ranging between $5 \%$ and $90 \%$ of each subject's maximum force capability. We anticipated that reaction time would decrease with increments of rate of force production in a manner relatively independent of force level and duration.

\section{METHOD}

\section{Subjects}

The subjects were 3 student volunteers. There were 2 males and 1 female. Each subject was right handed. 


\section{Apparatus}

The apparatus consisted of a horizontal arm extension device that was made up of a rigidly constructed table and a forearm extension lever and seating apparatus. The forearm extension lever consisted of a $3 / 4$ in. $(1.9-\mathrm{cm})$ steel bar that was bent at a $90^{\circ}$ angle. The lower portion of the bar, $60 \mathrm{~cm}$ in length, was mounted to the vertical face of the table by two sets of bearings. The upper portion of the bar, $47 \mathrm{~cm}$ in length, was parallel to and $3 \mathrm{~cm}$ above the table top. The two sets of bearings allowed the bar to be rotated about its vertical axis. Also attached to the table, next to the arm bar, was a seating device that could be adjusted to accommodate subjects of differing heights. Restraining straps were attached to the table and the seating device in a position that allowed them to be placed around the subject's waist and chest to stabilize the subject. The entire assembly was firmly anchored to the wall of the testing room, allowing for a rigid structure that was required for the higher levels of force production.

A piezoelectric force transducer (PCB, Piezotronics, Inc.) was used to measure the force produced against the forearm extension lever. The force transducer was attached at one end to the distal portion of the horizontal segment of the extension lever and at the other end to the wall of the testing room with a rigid bar. The angle formed by the extension bar and the force transducer was $90^{\circ}$. The force produced by subjects against the extension bar was obtained from the output of the force transducer. The signal from the force transducer was sampled at $1000 \mathrm{~Hz}$ by a DEC PDP-11/23 computer. A Tektronix 4010 graphics terminal provided subjects with the force-time profile of their responses.

\section{Procedure}

The subject was seated on the adjustable chair of the apparatus, and the seat height was adjusted so that when the subject placed his/her right arm on the horizontal section of the forearm extension lever, the upper arm was parallel to the ground. The subject's elbow was positioned over the vertical segment of the bar with his/her hand placed immediately behind the force transducer. The subject grasped the bar and attempted to produce force by extending the forearm at the elbow. Force was produced isometrically because the lever and force transducer were fastened to the wall of the testing room by a rigid bar.

There were two phases of the experiment. In the initial phase, on Day 1, each subject performed 25 trials in each of three conditions. The subjects were instructed to produce their maximum possible force while maintaining a prescribed time to peak force. The times to peak force were 100,200 , and $400 \mathrm{msec}$. The subjects were given feedback as to the time to peak force following each trial. Based upon these data, the average maximum force obtained with a time to peak force of 100,200 , and $400 \mathrm{msec}$ was calculated.

The second phase consisted of conditions in which each subject attempted to produce a force output with a specified peak force and time to peak force value. Two conditions were tested per day over 12 consecutive days. The experimental protocol was identical regardless of the experimental condition. The subject was presented with a warning light that was followed by a stimulus light with a random foreperiod between 1 and $3 \mathrm{sec}$. Upon presentation of the stimulus light, the subject was instructed to produce the designated force-time curve with a minimum reaction time delay. Approximately $8 \mathrm{sec}$ after the completion of the response, a representation of the just-completed response and a force-time template were shown on the Tektronix screen. The force-time template was Gaussian in form and closely resembled the shape of the force-time curve normally produced by subjects. The height of the template was a function of the level of peak force for the condition, and the duration was a function of the time to peak force. The duration of the force template was two times the time to peak force. The actual force-time curve produced by the subject for a trial and the template were aligned at peak force to facilitate comparison. The subject was instructed to use the feedback to ensure that the designated forcetime profile was produced. A few seconds after presentation of the forcetime profiles, the warning light was presented to start a new trial.

The average maximum peak force value for each of the three time to peak force conditions obtained on Day 1 were used to determine the specific force-time conditions for each subject. Peak force values representing $5 \%, 10 \%, 15 \%, 25 \%, 50 \%, 75 \%, 85 \%$, and $90 \%$ of the maximum peak force for each time to peak force were used. Each subject had different maximum force values; therefore, the specific peak force levels were different for each subject. Thus there were 24 conditions for each subject ( 8 percentages of maximum peak force $\times 3$ times to peak force). Each condition consisted of 80 trials with an intertrial interval of $20 \mathrm{sec}$. The short force durations and the long intertrial interval reduced any fatigue effects. Time to peak force was blocked so that the subjects received all conditions at one time to peak force before changing to a new time to peak force. Trials were also blocked so that the subjects received 80 consecutive trials at each percentage of peak force. The order of times to peak force and percentages of peak force within a time to peak force was determined randomly.

\section{RESULTS}

The results from the maximum force trials obtained on Day 1 indicated that maximum peak force increased systematically with increments of time to peak force. The mean maximum force produced by the three subjects ranged between 47 and $68 \mathrm{~N}(100 \mathrm{msec}), 54$ and $100 \mathrm{~N}$ (200 msec, and 60 and $120 \mathrm{~N}(400 \mathrm{msec})$. These findings are consistent with the proposal that maximum peak force increases at a negatively accelerating rate with increments of time to peak force (Newell \& Carlton, 1985).

The remaining analyses were conducted over the last 50 trials in each force-time condition to eliminate trials in which the subject was learning to produce the designated force-time profile. The data for time to peak force and peak force level indicated that the subjects, on average, matched the criterion force-time pattern at each of the conditions. The deviations from the criterion values were always less than $10 \%$.

The mean reaction times, as a function of time to peak force and the percentage of maximum force, are shown in Figure 1. As force level increased, the reaction time decreased $[F(7,14)=8.68, p<.01]$; however, there was no significant effect for movement duration $(F<1)$. Although the 100 -msec time to peak force condition had faster reaction times at the low and high percentage of maximum conditions, faster times were not demonstrated at intermediate values. Generally, shorter duration

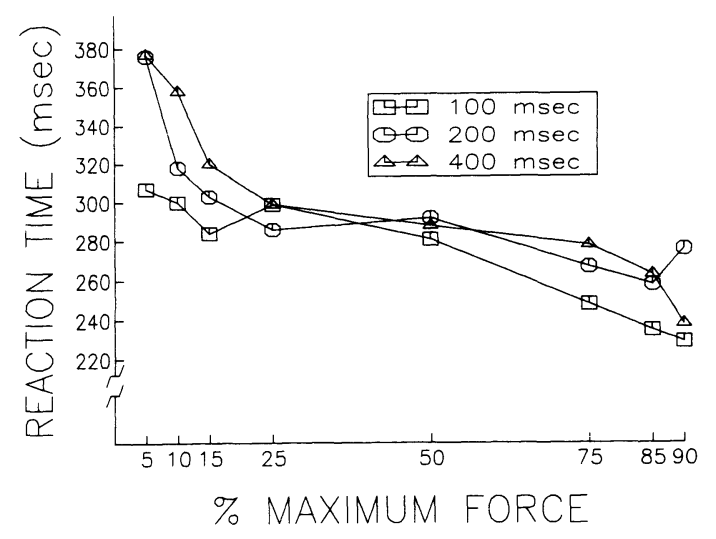

Figure 1. Reaction time (msec) as a function of the time to peak force and percentage of maximum peak force produced. 


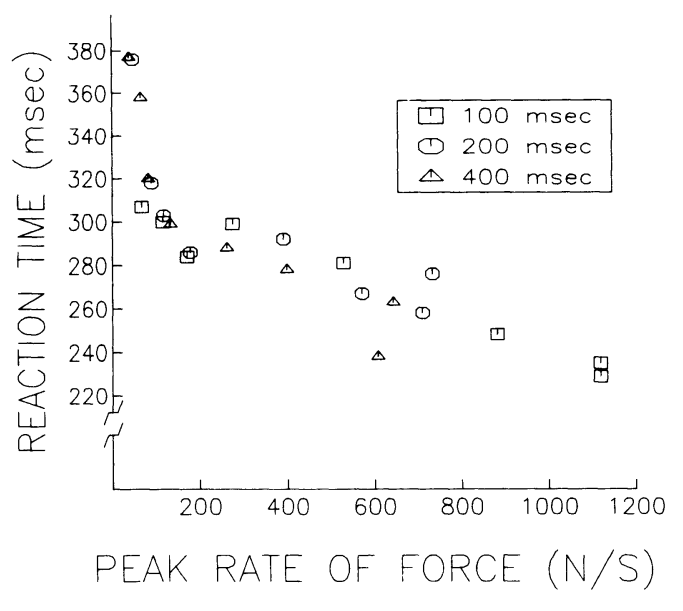

Figure 2. Reaction time (msec) for each of the 24 peak force/time to peak force conditions as a function of the peak rate of force production.

responses had faster reaction times at low percentage of maximum forces, but this did not generalize across the range of forces. Given the small number of subjects, it is not unusual to see some variability even in the averaged data. However, given the range of the forces and durations employed, we expected a strong relation between response characteristics and reaction time.

To further examine the relationship between reaction time and response dynamics, we reanalyzed the results and plotted reaction time as a function of the peak rate of force production for each force-time condition (L. G. Carlton et al., 1987). The actual force level produced by each subject varied due to differences in each person's maximum force capabilities; therefore, the average rate of force production was different for each condition. Figure 2 provides the average reaction time for the 24 force-time conditions plotted as a function of the peak rate of force. As the rate of force production increased, the reaction time systematically decreased. The form of the function appears to be curvilinear, with greater decreases in reaction time associated with low peak rates of force production. There are two points on the reaction time curve that are somewhat deviant from the general trend. In each case, these data points are from the $90 \%$ of maximum force condition. It may be that this condition had some effect on the strategies employed by the subjects because of the near maximal forces required.

\section{DISCUSSION}

The notion that physical constraints related to response kinetics influence the observed reaction time for any given trial dates back at least to DeLabarre et al. (1897). A number of experiments since that study have examined the link between reaction time and force production, but these studies have been limited with respect to the range of forces examined and by the instructional sets given to subjects. The current findings clearly show that reaction time is independent of response duration and decreases at a negatively accelerating rate with increments of rate of force production.
This relationship between reaction time and response dynamics has also been shown in a number of experiments in which the average velocity of discrete movements was manipulated (M. J. Carlton et al., 1985; Falkenberg \& Newell, 1980; Newell, Hoshizaki, Carlton, \& Halbert, 1979). When reaction time is plotted as a function of peak rate of acceleration, which is proportional to peak rate of force production, a similar curvilinear relationship between reaction time and peak rate of force production is obtained (M. J. Carlton et al., 1985). It should be noted that the reduction in reaction time at higher rates of force production cannot be attributable solely to the electromechanical delay of muscle. Fractionated reaction time measures of premotor and motor time each display a similar curvilinear trend when plotted against peak rate of force production (L. G. Carlton et al., 1987; M. J. Carlton et al., 1985).

There are many factors other than the rate of force production that influence reaction time (Klapp, 1981). However, any response programming interpretation of reaction time must consider the force production characteristics of the response. The relationship between force production characteristics and reaction time appears to be independent of the classification of the movement, and is consistent across isometric and discrete movement tasks.

Although measures of reaction time have traditionally been used as an indication of the amount of time necessary to generate a response program (Henry \& Rogers, 1960), there has been considerable debate over the type of experimental paradigm that is appropriate for examining response programming. Henry (1980) has continued to argue that response programming occurs after stimulus presentation, whereas Klapp (1981) has argued that response programming must be evaluated using choice reaction time procedures in order to minimize prestimulus programming. The present data do not speak to the motor programming paradigm issue, but they do indicate that programming interpretations based on reaction time data must be made with some caution. The relationship between reaction time and response dynamics found in this study is independent of programming considerations and suggests that there is a natural relationship between reaction time and response dynamics.

\section{REFERENCES}

Carlton, L. G., Carlton, M. J., \& Newell, K. M. (1987). Reaction time and response dynamics. Quarterly Journal of Experimental Psychology, 39A, 337-360.

Carlton, M. J., Robertson, R. N., Carlton, L. G., \& Newell, K. M. (1985). Response timing variability: Coherence of kinematic and EMG parameters. Journal of Motor Behavior, 17, 301-319.

Delabarre, E. B., Logan, R. R., \& Reed, A. Z. (1897). The force and rapidity of reaction movements. Psychological Review, 4, 615-631.

FalkenberG, L. E., \& Newell, K. M. (1980). Relative contribution of movement time, amplitude, and velocity to response initiation. Journal of Experimental Psychology: Human Perception \& Performance, 6, 760-768.

GHEZ, C., \& ViCARIO, D. (1978). The control of rapid limb movement in the cat: I. Response latency. Experimental Brain Research, 33, 173-189.

HENRY, F. M. (1980). Use of simple reaction in motor programming studies: A reply to Klapp, Wyatt, \& Lingo. Journal of Motor Behavior, 12, 237-242.

HENRY, F. M. (1981). The evolution of the memory drum theory of neuromotor reaction. In G. A. Brooks (Ed.), Perspectives on the academic discipline of physical education (pp. 301-322). Champaign, IL: Human Kinetics.

HenRY, F. M., \& Rogers, E. E. (1960). Increased response latency for complicated movements and a "memory drum" theory of neuromotor reaction. Research Quarterly, 31, 448-458.

KLAPP, S. T. (1977). Reaction time analysis of programmed control of aimed movements. In R. S. Hutton (Ed.), Exercise and sport sciences reviews (Vol. 5, pp. 231-253). Santa Barbara, CA: Journal Publishing Affiliates.

KLAPP, S. T. (1981). Motor programming is not the only process which can influence RT: Some thoughts on the Marteniuk and MacKenzie analysis. Journal of Motor Behavior, 13, 320-328.

KLAPP, S. T., \& ERWIN, C. I. (1976). Relation between programming 
time and duration of the response being programmed. Journal of Experimental Psychology: Human Perception \& Performance, 2, 591-598.

KlaPP, S. T., McRaE, J., \& Long, W. (1978). Response programming vs. alternative interpretations of the "dit-dah" reaction time effect. Bulletin of the Psychonomic Society, 11, 5-6.

KlaPP, S. T., WyatT, E., \& Lingo, W. (1974). Response programming in simple and choice reactions. Journal of Motor Behavior, 6 , 263-271.

KLEMMER, E. T. (1957). Rate of force application in a simple reaction time test. Journal of Applied Psychology, 41, 329-332.

Kornblum, S., \& ReQuin, J. (Eds.). (1984). Preparatory states and processes. Hillsdale, NJ: Erlbaum.

Nagasaki, H., Aoki, F., \& Nakamura, R. (1983). Premotor and motor reaction time as a function of force output. Perceptual \& Motor Skills, 57, 859-867.

Newell, K. M., \& Cartton, L. G. (1985). On the relationship be- tween force and force variability in isometric tasks. Journal of Motor Behavior, 17, 230-241.

Newell, K. M., Hoshizaki, L. E. F., Carlton, M. J., \& Halbert, J. A. (1979). Movement time and velocity as determinants of movement timing accuracy. Journal of Motor Behavior, 11, 49-58.

Sternberg, S., Monsell, S., Knoll, R. L., \& Wright, C.E. (1978). The latency and duration of rapid movement sequences: Comparisons of speech and typewriting. In G. E. Stelmach (Ed.), Information processing in motor control and learning (pp. 117-152). New York: Academic Press.

WoODWORTH, R. S. (1938). Experimental psychology. New York: Henry Holt.

Zelaznik, H. N., Shapiro, D. C., \& Carter, M. C. (1982). The specification of digit and duration during motor programming: A new method of precuing. Journal of Motor Behavior, 14, 57-68.

(Manuscript received for publication March 28, 1987.) 